\title{
The Prevalence and Implication of Zinc Deficiency in Patients With Chronic Liver Disease
}

\author{
Kazuhiro Katayama ${ }^{a, v}$, Takumi Kawaguchi ${ }^{b}$, Koichi Shiraishic ${ }^{c}$, Toshifumi Ito ${ }^{d}$, Kazutomo Suzukie, \\ Chizu Koreeda ${ }^{\mathrm{f}}$, Takaaki Ohtake ${ }^{\mathrm{g}}$, Motoh Iwasa $^{\mathrm{h}}$, Yoshio Tokumoto ${ }^{\mathrm{i}}$, Ryujin Endo ${ }^{\mathrm{j}}$, Naohiro Kawamura ${ }^{\mathrm{k}}$, \\ Makoto Shiraki ${ }^{1}$, Tatsunori Hanai ${ }^{1}$, Daiki Habu ${ }^{\mathrm{m}}$, Satoru Tsuruta ${ }^{\mathrm{n}}$, Hironori Sakai ${ }^{\mathrm{n}}$, Yoshiyuki Miwa ${ }^{\mathrm{o}}$, \\ Norifumi Kawadap ${ }^{p}$, Akinobu Katoq, Yoshiyuki Takei ${ }^{\mathrm{h}}$, Tetsuya Mine ${ }^{\mathrm{c}}$, Yutaka Kohgog, r, Toshihito Sekif \\ Michio Sata ${ }^{\mathrm{b}}$, Yuri Ito ${ }^{\mathrm{s}}$, Keisuke Fukui ${ }^{\mathrm{s}}$, Shuhei Nishiguchi ${ }^{\mathrm{t}}$, Hisataka Moriwaki ${ }^{1}$, Kazuyuki Suzuki ${ }^{u}$
}

Manuscript submitted January 30, 2018, accepted February 20, 2018

\section{Abstract}

Background: Patients with liver cirrhosis often exhibit zinc deficiency. Although zinc is involved in many bioactivities, many aspects of clinical implications of zinc deficiency in liver cirrhosis remain unclear. We aimed to reveal the prevalence and implications of zinc deficiency in liver cirrhosis by assessing associations with parameters such as clinical symptoms and laboratory data.

Methods: In 235 cirrhosis patients enrolled at multiple medical institutions in 2009, we assessed how blood zinc levels were associated with their clinical symptoms, patients characteristics, and liver function test results.

Results: Blood zinc levels were most strongly correlated with blood albumin levels among the study parameters $(\mathrm{r}=0.587, \mathrm{P}<0.0001)$. When blood albumin levels were $\leq 3.5 \mathrm{~g} / \mathrm{dL}$, blood zinc levels were $<70 \mu \mathrm{g} / \mathrm{dL}$ in $88 \%$ of patients. Additionally, significant correlations were observed with age $(\mathrm{r}=-0.253, \mathrm{P}=0.0014)$, aspartate aminotransferase levels $(r=-0.254, P=0.0020)$, total bilirubin levels $(r=-0.222$, $\mathrm{P}=0.0053)$, prothrombin time $(\mathrm{r}=-0.255, \mathrm{P}=0.0029)$, branchedchain amino acid to tyrosine ratio $(\mathrm{r}=0.357, \mathrm{P}<0.0001)$, Child-Pugh score $(\mathrm{r}=0.469, \mathrm{P}<0.0001)$, ammonia levels $(\mathrm{r}=-0.246, \mathrm{P}=0.0028)$, and total cholesterol levels $(\mathrm{r}=0.314, \mathrm{P}<0.0001)$. Blood zinc levels were significantly lower in patients with edema/ascites $(\mathrm{P}<0.0001)$, those with hepatic encephalopathy $(\mathrm{P}=0.0215)$, those receiving oral diuretics $(\mathrm{P}=0.0045)$, and those receiving oral branched-chain amino acids $(\mathrm{P}<0.0001)$ than in those without these conditions.

Conclusions: Zinc deficiency is prevalent in cirrhosis patients, whereas nitrogen metabolic disorders, particularly hypoalbuminemia, can be an indicator of zinc deficiency. Thus, cirrhosis patients exhibiting a nitrogen metabolic disorder should be examined for the presence of zinc deficiency.

Keywords: Liver cirrhosis; Nitrogen metabolism; Branched-chain amino acid; Ammonia; Albumin

\section{Introduction}

While the liver is the primary organ for metabolism of nutri- 
ents, liver cirrhosis causes protein and energy metabolic disorders, which contribute to poor prognosis or the development of various complications including hepatic encephalopathy [1-6]. Several studies have shown that nutritional interventions are effective for patients with liver cirrhosis who exhibit nutritional and metabolic disorders [7-10]. It has been demonstrated that treatment of liver cirrhosis with branched-chain amino acids (BCAAs) relieves nutritional and metabolic dysfunction, prevents the development of complications and liver cancer, and improves prognosis. Furthermore, among many trace elements existing in the body, zinc has recently been shown to be deeply associated with the pathology of liver cirrhosis [11-14].

Zinc is an essential trace element with various biological effects [13]. As more than 300 proteins contain domains with zinc and these domains are important for regulating cellular functions, zinc plays an important role in cell growth, differentiation, apoptosis and metabolism [12, 15-19]. Zinc homeostasis is primarily preserved by a balance between the zincbinding protein metallothionein and the expression of two zinc transporters [12, 20-24]. There were some reports that zinc deficiency resulted in numerous problems, including growth disorder, cognitive disorder, and compromised immune function [25-28]. Zinc deficiency occurs as a result of nutritional factors, but also in various disease states (malabsorption, Crohn's disease, alcoholism, liver cirrhosis, chronic renal disease, and other chronically debilitating diseases) [13]. Factors that are potentially responsible for zinc deficiency in liver cirrhosis include disturbed zinc absorption by the digestive tract and increased zinc excretion in the urine $[12,13]$. The effects of cytokines, mainly interleukin-6 and endotoxins also contributed zinc deficiency [12]. Furthermore, diuretics aggravate zinc deficiencies in patients with liver cirrhosis by increasing zinc excretion in the urine [29].

Several studies showed a statistically significant inverse relationship between the serum levels of zinc and ammonia, and that zinc deficiency is related to the pathogenesis of hepatic encephalopathy [30-32]. On the basis of these findings, there were some reports which examined the effects of zinc supplementation in liver cirrhosis with hyperammonemia [30, 33-36]. Marchesini et al showed that 3-month supplementation of zinc in patients with hepatic encephalopathy reduced serum ammonia levels, but this was not a randomized-controlled trial [14]. Two randomized-controlled trials of rather short period ( 8 and 10 days) have been performed to examine these effects, and the results were controversial $[33,37]$. Recently, we performed a longer period randomized, placebo-controlled double-blind trial, and indicated that zinc supplementation for 3 months seems effective and safe for treating hyperammonemia in liver cirrhosis [38].

The main effect of zinc supplementation on reducing serum levels of ammonia proposed thus far is increased activity of ornithine transcarbamylase, a key enzyme of the urea cycle in the liver [13]. Riggio $\mathrm{O}$ et al showed that liver ornithine transcarbamylase activity decreased and plasma ammonia level increased in zinc-deficient rats, while the activity of the enzyme significantly increased in zinc-supplemented cirrhotic rats [34].

Because zinc has been revealed to play an important role in the synthesis, storage, and secretion of insulin, zinc deficiency affects not only insulin secretion but also insulin re- sistance [39-41]. In diabetes mellitus, zinc deficiency has been found to be associated with glucose tolerance and the development of complications of diabetes mellitus, whereas there are some reports that zinc supplementation improves glucose tolerance and reduces the incidence of complications [42, 43]. Although glucose intolerance is also prevalent in liver cirrhosis, the association between glucose intolerance and zinc in liver cirrhosis has been little studied.

These previous studies have increasingly shown that zinc deficiency is associated with nitrogen metabolic disorders, mainly affecting ammonia in liver cirrhosis, and that zinc supplementation is effective for patients with these conditions. However, not all patients with liver cirrhosis exhibit zinc deficiency. In addition to nitrogen metabolic disorders, other metabolic disorders, such as glucose intolerance, often coexist with liver cirrhosis. A large-scale study on the prevalence of zinc deficiency in liver cirrhosis and the associations between zinc deficiency and various pathological conditions of liver cirrhosis may provide answers to the questions of which cirrhosis patients should be suspected of having zinc deficiency and for which cirrhosis patients should zinc supplementation be considered. We previously conducted a prospective study enrolling 299 cirrhosis patients to assess the effects of BCAA therapy. Using data obtained in this previous study, we investigated the prevalence of zinc deficiency in liver cirrhosis and the association between zinc deficiency and clinical symptoms in the present study.

\section{Patients and Methods}

\section{Patients}

In 2009,299 cirrhosis patients without hepatocellular carcinoma were enrolled from 14 medical institutes in Japan. The diagnosis of liver cirrhosis was based on an aspartate aminotransferase (AST)-to-platelet ratio index $>1.5$; morphologic changes of the liver such as hypertrophy of the left lateral and caudate lobes or atrophy of the right posterior hepatic lobe on ultrasonography, computed tomography, and/or magnetic resonance imaging; or a pseudo-lobule formation finding on histopathologic examination.

Of these 299 patients, 235 who had not received any oral zinc preparations were included in the present study. In addition to age and sex, the following clinical data were collected: cause of liver diseases; the presence or absence of current or prior history of hepatic encephalopathy, ascites, and edema; the presence or absence of the use of diuretics and BCAAs; and the presence or absence of treatment for diabetes mellitus (Table 1).

\section{Methods}

Correlations of blood zinc concentrations with patients' characteristics and liver function test results

After overnight fasting, blood samples were obtained from 
Table 1. Patients' Characteristics

\begin{tabular}{|c|c|}
\hline $\mathrm{N}$ & 235 \\
\hline Age $(y / o)$ & $67.4 \pm 11.1$ \\
\hline Gender (male/female) & $109 / 126$ \\
\hline Etiology (HCV/HBV/alcohol/NASH/others) & $148 / 27 / 25 / 15 / 20$ \\
\hline BMI $\left(\mathrm{kg} / \mathrm{m}^{2}\right)$ & $23.8 \pm 4.1$ \\
\hline Daily alcohol intake (none $/<60 \mathrm{~g} / \geq 60 \mathrm{~g}$ ) & $179 / 25 / 23$ \\
\hline Platelets count $\left(\times 10^{3} / \mathrm{mm}^{3}\right)$ & $102.9 \pm 64.4$ \\
\hline AST (IU/L) & $52.0 \pm 32.1$ \\
\hline $\operatorname{ALT}(\mathrm{IU} / \mathrm{L})$ & $41.8 \pm 29.4$ \\
\hline Albumin (g/dL) & $3.67 \pm 0.53$ \\
\hline Total bilirubin (mg/dL) & $1.10 \pm 0.53$ \\
\hline Prothrombin time (international normalized ratio) & $1.13 \pm 0.18$ \\
\hline Creatinine (mg/dL) & $0.90 \pm 1.30$ \\
\hline BUN (mg/dL) & $16.4 \pm 10.8$ \\
\hline BCAA-to-tyrosine ratio (BTR) & $3.97 \pm 1.77$ \\
\hline Child-Pugh score & $6.03 \pm 1.15$ \\
\hline Ammonia $(\mu \mathrm{g} / \mathrm{dL})$ & $48.7 \pm 33.5$ \\
\hline Total cholesterol (mg/dL) & $157.9 \pm 33.5$ \\
\hline Triglycerides (mg/dL) & $93.4 \pm 49.6$ \\
\hline Fasting blood glucose (mg/dL) & $114.8 \pm 31.7$ \\
\hline HbAlc (\%) & $5.50 \pm 1.03$ \\
\hline HOMA-IR & $5.71 \pm 8.86$ \\
\hline $\operatorname{Zinc}(\mu \mathrm{g} / \mathrm{dL})$ & $64.1 \pm 15.8$ \\
\hline Ferritin (ng/mL) & $168.2 \pm 522.0$ \\
\hline $\operatorname{AFP}(\mathrm{ng} / \mathrm{mL})$ & $18.1 \pm 37.4$ \\
\hline Edema, ascites (+/-) & $46 / 187$ \\
\hline Administration of diuretics (+/-) & $78 / 157$ \\
\hline Administration of BCAA $(+/-)$ & $78 / 157$ \\
\hline Administration of oral DM agents (+/-) & $56 / 179$ \\
\hline Administration of insulin (+/-) & $22 / 213$ \\
\hline
\end{tabular}

Values are expressed as mean $\pm \mathrm{SD}$. y/o: years old; HCV: hepatitis $C$ virus; HBV: hepatitis B virus; NASH: non-alcoholic steatohepatitis; BMI: body mass index; AST: aspartate aminotransferase; ALT: alanine aminotransferase; BUN: blood urea nitrogen; BCAA: branched-chain amino acid; HOMA-IR: homeostasis model assessment-insulin resistance; AFP: alpha-fetoprotein; DM: diabetes mellitus.

the antecubital vein in the morning. The association between serum zinc and patients' characteristics or liver function tests was analysed by the statistical method described in the following section.

\section{Statistical analysis}

The correlation between blood zinc levels and continuous variables was analysed using linear regression. An absolute $r$ value of 0.2 to less than 0.4 was considered to indicate a weak correlation, that of 0.4 to less than 0.7 a correlation, and that of less than 0.2 no correlation. Regarding the correlation between blood zinc levels and categorical variables, intergroup significant differences in blood zinc levels were tested using the Mann-Whitney test for two-level categorical variables and by the Kruskal-Wallis test for more than three-level categorical variables. A P value of less than 0.05 was considered to indicate a significant difference, in other words, an association with blood zinc levels.

\section{Ethics}

This study protocol conformed to the ethical guidelines of the 1975 Declaration of Helsinki as reflected in the prior approval given by the institutional review board of each institution.

\section{Results}

\section{Association of blood zinc concentrations with patient char- acteristics and laboratory data}

Blood zinc concentrations were correlated with blood albumin levels and Child-Pugh scores, whereas they were weakly correlated with age, AST, prothrombin time, BCAA-to-tyrosine ratio (BTR), blood ammonia levels, and total cholesterol levels (Table 2) (Fig. 1). Among the categorical variables, blood zinc levels showed significant intergroup differences in cause of liver disease, the presence or absence of edema/ascites and hepatic encephalopathy, and the use of diuretics and BCAAs. These variables were found to be associated with blood zinc levels (Table 3).

\section{Blood albumin levels and the probability of zinc deficiency}

Because the strongest correlation was observed between blood albumin and blood zinc levels, the probability of zinc deficiency was assessed according to blood albumin levels (Fig. 2). In patients diagnosed with liver cirrhosis, the probability of having a blood zinc level of less than $70 \mu \mathrm{g} / \mathrm{dL}$ was $79 \%$ with a blood albumin level of $4.0 \mathrm{~g} / \mathrm{dL}$ or lower and $88 \%$ with a blood albumin level of $3.5 \mathrm{~g} / \mathrm{dL}$ or lower.

\section{Discussion}

In the present study, many cirrhosis patients exhibited hypozincemia, whereas blood zinc levels were associated with indicators of nitrogen metabolism, mainly blood albumin levels, as well as several blood test parameters, clinical symptoms, and the presence or absence of the use of oral medication. Particularly, blood albumin levels were strongly associated with blood zinc levels. Thus, hypoalbuminemia detected in cirrhosis patients can be a useful indicator of zinc deficiency.

It cannot be concluded from the present study alone 
Table 2. Relationship Between Serum Zinc and Patients' Characteristics (Continuous Variables)

\begin{tabular}{lll}
\hline & $|\mathbf{r}|$ & P value \\
\hline Age $(\mathrm{y} / \mathrm{o})$ & 0.253 & 0.0014 \\
BMI $\left(\mathrm{kg} / \mathrm{m}^{2}\right)$ & 0.158 & 0.067 \\
Platelets count $\left(\times 10^{3} / \mathrm{mm}^{3}\right)$ & 0.189 & 0.0177 \\
AST $(\mathrm{IU} / \mathrm{L})$ & 0.245 & 0.002 \\
ALT $(\mathrm{IU} / \mathrm{L})$ & 0.077 & 0.3384 \\
Albumin $(\mathrm{g} / \mathrm{dL})$ & 0.587 & $<0.0001$ \\
Total bilirubin $(\mathrm{mg} / \mathrm{dL})$ & 0.222 & 0.0053 \\
Prothrombin time (international normalized ratio) & 0.255 & 0.0029 \\
Creatinine (mg/dL) & 0.149 & 0.0688 \\
BUN (mg/dL) & 0.128 & 0.1231 \\
BCAA-to-tyrosine ratio (BTR) & 0.357 & $<0.0001$ \\
Child-Pugh score & 0.469 & $<0.0001$ \\
Ammonia $(\mu \mathrm{g} / \mathrm{dL})$ & 0.246 & 0.0028 \\
Total cholesterol (mg/dL) & 0.314 & $<0.0001$ \\
Triglycerides (mg/dL) & 0.079 & 0.3493 \\
Fasting blood glucose (mg/dL) & 0.076 & 0.3527 \\
HbA1c $(\%)$ & 0.115 & 0.1623 \\
HOMA-IR & 0.072 & 0.4165 \\
Ferritin (ng/mL) & 0.069 & 0.4146 \\
AFP (ng/mL) & 0.17 & 0.0344 \\
\hline
\end{tabular}

y/o: years old; BMI: body mass index; AST: aspartate aminotransferase; ALT: alanine aminotransferase; BUN: blood urea nitrogen; BCAA: branched-chain amino acid; HOMA-IR: homeostasis model assessment-insulin resistance; AFP: alpha-fetoprotein; DM: diabetes mellitus. whether these variables correlated with blood zinc levels are a cause or outcome of zinc deficiency. This issue is discussed along with previously reported studies in the following section (Fig. 3).

Blood albumin levels, blood ammonia levels, BTR, and prothrombin time, which were correlated with blood zinc levels, are all blood test parameters reflecting protein metabolism. As described above in the Introduction, zinc is involved in the function of the hepatic urea cycle, and zinc deficiency causes hyperammonemia through impaired ammonia metabolic capacity due to hypofunction of the urea cycle $[6,14]$. When ammonia that is not metabolised in the urea cycle is metabolised by glutamine synthetase present in skeletal muscles and other organs, BCAAs are consumed and become deficient, which decreases the BTR. BCAA deficiency, which reduces the protein synthetic capacity, ultimately leads to decreased blood albumin levels $[6,44]$. Moreover, the present study revealed that blood zinc levels were significantly lower in patients with a current or prior history of hepatic encephalopathy. These findings, on the whole, support that zinc deficiency may be involved in the development of hepatic encephalopathy because zinc deficiency impairs ammonia metabolism, subsequently causing hyperammonemia. Among the parameters reflecting nitrogen metabolism, blood albumin levels were most strongly correlated with blood zinc concentrations. As described above, while zinc deficiency may contribute to decreased blood albumin levels, hypoalbuminemia leads to decreased blood zinc concentrations because zinc binds mainly to albumin and is transported in blood [13]. In other words, zinc deficiency is a cause as well as an outcome of hypoalbuminemia. Thus, because of the strong association between blood zinc and blood albumin levels, a strong correlation can be assumed to exist between them. Because several studies have revealed that such nitrogen metabolic disorders and hepatic encephalopathy are
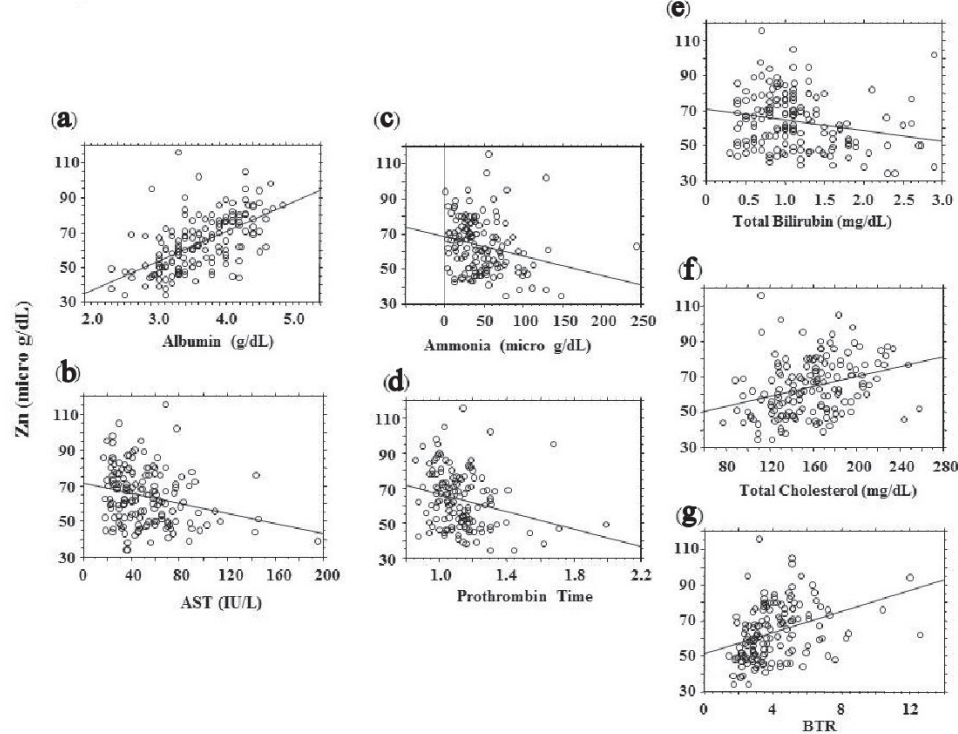

Figure 1. Correlations between blood zinc concentrations (vertical axis) and blood test results (absolute $r \geq 2.0$ ). (a) Albumin. (b) Aspartate aminotransferase (AST). (c) Ammonia. (d) Prothrombin time. (e) Total bilirubin. (f) Total cholesterol. (g) BCAA-totyrosine ratio (BTR). 
Table 3. Relationship Between Serum Zinc and Patients' Characteristics (Categorized Variables)

\begin{tabular}{lll}
\hline & Serum level of zinc $(\mu \mathrm{g} / \mathbf{d L})$ & P value \\
\hline Gender (male/female) & $65.7 \pm 16.3 / 62.6 \pm 15.3$ & 0.2423 \\
Etiology $(\mathrm{C} / \mathrm{B} / \mathrm{Al} / \mathrm{N} / \mathrm{O})$ & $62.2 \pm 14.9 / 72.9 \pm 14.3 / 66.1 \pm 20.5 / 60.8 \pm 13.7 / 60.9 \pm 14.8$ & 0.04 \\
Daily alcohol intake $($ none $/<60 \mathrm{~g} / \geq 60 \mathrm{~g})$ & $62.3 \pm 15.0 / 73.0 \pm 19.3 / 63.4 \pm 16.1$ & 0.1132 \\
Edema or ascites $(+/-)$ & $56.0 \pm 19.6 / 65.9 \pm 14.3$ & $<0.0001$ \\
Hepatic encephalopathy $(+/-)$ & $51.3 \pm 18.3 / 64.8 \pm 15.4$ & 0.0215 \\
Administration of diuretics (+/-) & $58.6 \pm 18.9 / 65.6 \pm 14.6$ & 0.0045 \\
Administration of BCAA (+/-) & $55.0 \pm 13.1 / 70.5 \pm 14.4$ & $<0.0001$ \\
Administration of oral DM agents $(+/-)$ & $65.0 \pm 16.4 / 63.8 \pm 15.7$ & 0.7637 \\
Administration of insulin $(+/-)$ & $61.9 \pm 19.3 / 64.3 \pm 15.5$ & 0.347 \\
\hline
\end{tabular}

Values are expressed as mean \pm SD. C: hepatitis C virus; B: hepatitis B virus; Al: alcohol; N: non-alcoholic steatohepatitis; O: others; BCAA: branched-chain amino acid; DM: diabetes mellitus.

relieved by zinc supplementation [13, 14, 38, 44], zinc deficiency appears to be the cause of such nitrogen metabolic disorders observed in liver cirrhosis.

Total bilirubin levels also showed a weak negative correlation with blood zinc concentrations. Regarding the association between bilirubin metabolism and zinc, there is a report indicating a negative correlation as observed in the present study [45]; however, the detailed mechanism remains unknown. Because zinc has been reported to suppress the enterohepatic circulation [46], it is possible that impairment of this function due to zinc deficiency contributes to increased blood bilirubin levels. However, given the small sample size, this issue needs to be investigated in the future.

AST levels also showed a weak negative correlation with blood zinc concentrations. Because zinc has been reported to have antioxidant and anti-inflammatory activities [13], zinc deficiency may be involved in aggravation of hepatocellular injury. However, the correlation coefficient was low, although there is also a report indicating that zinc levels were not correlated with either alanine aminotransferase or AST levels [45]. Thus, the association between hepatocellular injury and zinc also needs to be investigated in the future.

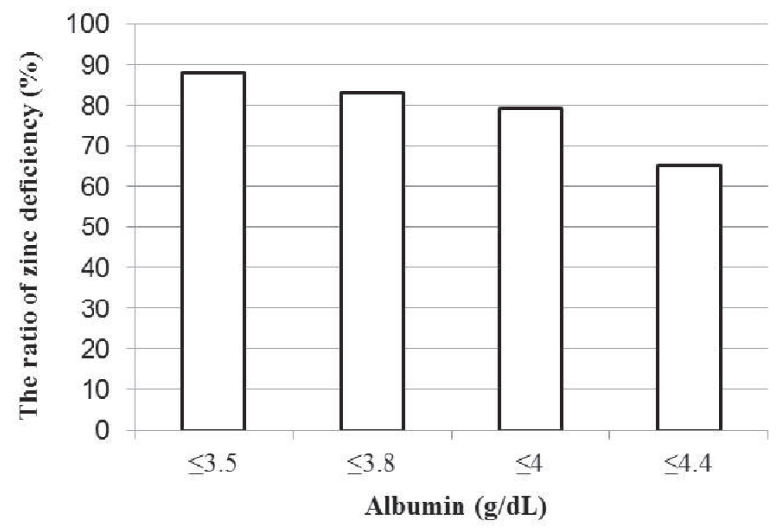

Figure 2. Blood albumin concentrations and the probability of zinc deficiency (blood zinc concentrations $<70 \mu \mathrm{g} / \mathrm{dL}$ ).
Blood cholesterol levels also showed a weak positive correlation with blood zinc levels. Regarding cholesterol metabolism, because zinc is involved in insulin secretion and insulin resistance, zinc deficiency has been reported to be a possible cause of increased cholesterol levels [47]. This finding, which differs from the results of the present study, indicates that zinc may play different roles in dyslipidaemia in people with and without liver cirrhosis.

In patients receiving diuretics or BCAAs, blood zinc concentrations were significantly lower. Whether the use of these drugs is a cause or outcome of zinc deficiency is discussed as follows. In Japan, because the use of BCAAs is recommended for patients with liver cirrhosis accompanied by hypoalbuminemia, BCAAs are administered to patients exhibiting hypoalbuminemia. Meanwhile, the use of diuretics is recommended for patients with liver cirrhosis accompanied by ascites or edema. However, because one of the causes of ascites and edema is generally hypoalbuminemia, diuretics are also highly likely to be administered in consequence of the hypoalbuminemia. However, because other studies have shown that the use of diuretics may increase urinary excretion of zinc [29, 48], the drugs may enhance zinc deficiency.

Regarding alcohol drinking, because previous studies have indicated that patients with alcoholic liver cirrhosis exhibit zinc deficiency, it has been suggested that alcohol drinking may cause zinc deficiency [13]. However, the present study did not show any correlation between alcohol consumption and blood zinc concentrations. In alcoholic liver cirrhosis, nitrogen metabolic disorders, such as hypoalbuminemia, are observed, for example, because of disease progression or malnutrition due to alcohol drinking. Thus, patients with alcoholic liver cirrhosis may have exhibited zinc deficiency. Further studies are needed to determine the causal relationship between alcohol drinking and zinc deficiency. Although blood zinc levels showed significant intergroup differences in cause of liver disease (Table 3), blood albumin levels also showed significant intergroup differences in cause of liver disease (HCV, $3.6 \pm 0.5$ g/dL; HBV, $4.0 \pm 0.6$; alcohol, $3.6 \pm 0.7$; non-alcoholic steatohepatitis (NASH), $3.8 \pm 0.7 ; \mathrm{P}=0.0203$ by Mann-Whitney). Therefore, it is likely that the intergroup difference in blood zinc levels was associated with the stage (function) of liver 


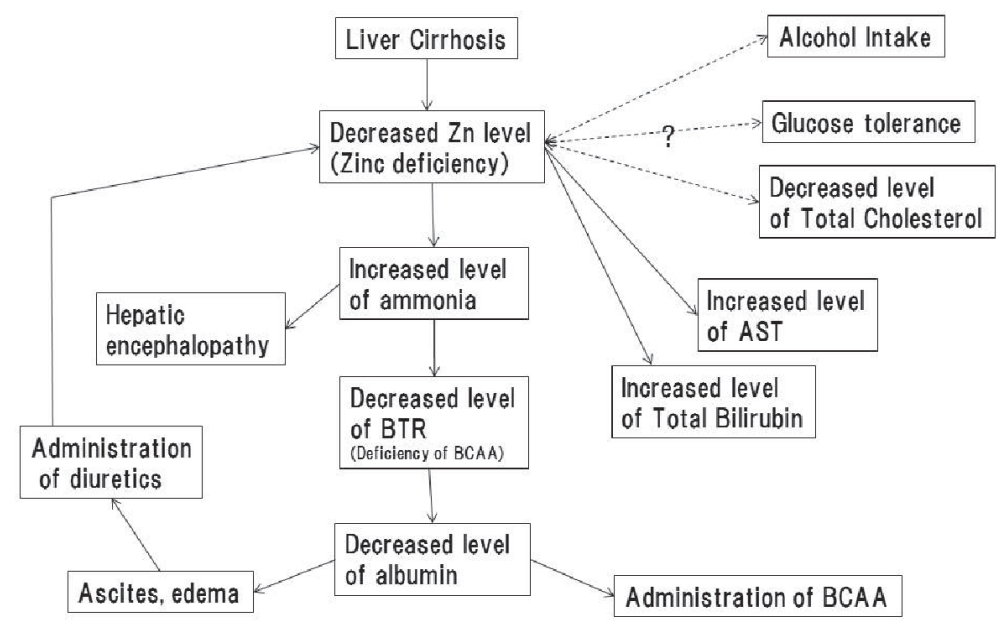

Figure 3. Hypotheses of causal relationships between zinc deficiency and pathological conditions of liver cirrhosis. The solid arrow indicates the presence of a causal relationship; dashed arrows indicate the causal relationship is unknown.

disease but not the cause.

In addition, no significant correlations were observed with glucose and lipid metabolism-related parameters, such as the presence or absence of abnormal glucose tolerance, the presence or absence of treatment of diabetes mellitus, and body mass index. Although several reports suggest that zinc is involved in insulin secretion and glucose tolerance [39-43], the present study does not prove any significant association between zinc and abnormal glucose tolerance due to liver cirrhosis.

The present study showed that blood zinc concentrations were correlated with many test parameters and clinical symptoms characteristic of liver cirrhosis. Particularly, zinc deficiency is involved in the pathological conditions of nitrogen metabolic disorders and hepatic encephalopathy. This supports the importance of zinc supplementation. As for the other parameters associated with blood zinc levels, the mechanisms of their associations need to be elucidated in the future. Moreover, because patients with hepatocellular carcinoma were excluded from the cirrhosis patients included in the present study, we did not assess the association between zinc and hepatocarcinogenesis. There is a report of a study suggesting the presence of this association [13], which is an important future issue to be addressed.

In conclusion, zinc deficiency is prevalent in liver cirrhosis, while many metabolic disorders, particularly hypoalbuminemia and other nitrogen metabolic disorders, are good indicators of zinc deficiency. Thus, the presence or absence of zinc deficiency should be evaluated in cirrhosis patients exhibiting nitrogen metabolic disorders.

\section{Conflict of Interest}

The authors declare that they have no conflict of interest.

\section{References}

1. Alberino F, Gatta A, Amodio P, Merkel C, Di Pascoli L,
Boffo G, Caregaro L. Nutrition and survival in patients with liver cirrhosis. Nutrition. 2001;17(6):445-450.

2. Christensen E, Schlichting P, Fauerholdt L, Gluud C, Andersen PK, Juhl E, Poulsen H, et al. Prognostic value of Child-Turcotte criteria in medically treated cirrhosis. Hepatology. 1984;4(3):430-435.

3. Tajika M, Kato M, Mohri H, Miwa Y, Kato T, Ohnishi H, Moriwaki $H$. Prognostic value of energy metabolism in patients with viral liver cirrhosis. Nutrition. 2002;18(3):229-234.

4. Trewby PN, Chase RA, Davis M, Williams R. The role of the false neurotransmitter octopamine in the hypotension of fulminant hepatic failure. Clin Sci Mol Med. 1977;52(3):305-310.

5. El-Serag HB, Tran T, Everhart JE. Diabetes increases the risk of chronic liver disease and hepatocellular carcinoma. Gastroenterology. 2004;126(2):460-468.

6. Katayama K. Ammonia and hepatic encephalopathy. Hepatol Res. 2004;30S:S71-S78.

7. Muto Y, Sato S, Watanabe A, Moriwaki H, Suzuki K, Kato A, Kato M, et al. Effects of oral branched-chain amino acid granules on event-free survival in patients with liver cirrhosis. Clin Gastroenterol Hepatol. 2005;3(7):705713.

8. Muto Y, Sato S, Watanabe A, Moriwaki H, Suzuki K, Kato A, Kato M, et al. Overweight and obesity increase the risk for liver cancer in patients with liver cirrhosis and longterm oral supplementation with branched-chain amino acid granules inhibits liver carcinogenesis in heavier patients with liver cirrhosis. Hepatol Res. 2006;35(3):204214.

9. Kawaguchi T, Shiraishi K, Ito T, Suzuki K, Koreeda C, Ohtake T, Iwasa $\mathrm{M}$, et al. Branched-chain amino acids prevent hepatocarcinogenesis and prolong survival of patients with cirrhosis. Clin Gastroenterol Hepatol. 2014;12(6):1012-1018 e1011.

10. Plank LD, Gane EJ, Peng S, Muthu C, Mathur S, Gillanders L, McIlroy K, et al. Nocturnal nutritional supplemen- 
tation improves total body protein status of patients with liver cirrhosis: a randomized 12-month trial. Hepatology. 2008;48(2):557-566.

11. Fargion S, Valenti L, Fracanzani AL. Hemochromatosis gene (HFE) mutations and cancer risk: expanding the clinical manifestations of hereditary iron overload. Hepatology. 2010;51(4):1119-1121.

12. Grungreiff $\mathrm{K}$, Reinhold $\mathrm{D}$, Wedemeyer $\mathrm{H}$. The role of zinc in liver cirrhosis. Ann Hepatol. 2016;15(1):7-16.

13. Stamoulis I, Kouraklis G, Theocharis S. Zinc and the liver: an active interaction. Dig Dis Sci. 2007;52(7):15951612.

14. Marchesini G, Fabbri A, Bianchi G, Brizi M, Zoli M. Zinc supplementation and amino acid-nitrogen metabolism in patients with advanced cirrhosis. Hepatology. 1996;23(5):1084-1092.

15. Prasad AS. Zinc: an overview. Nutrition. 1995;11(1 Sup$\mathrm{pl}): 93-99$.

16. Vallee BL, Falchuk KH. The biochemical basis of zinc physiology. Physiol Rev. 1993;73(1):79-118.

17. Pabo CO, Peisach E, Grant RA. Design and selection of novel Cys2His 2 zinc finger proteins. Annu Rev Biochem. 2001;70:313-340.

18. Joazeiro CA, Weissman AM. RING finger proteins: mediators of ubiquitin ligase activity. Cell. 2000;102(5):549552.

19. Kadrmas JL, Beckerle MC. The LIM domain: from the cytoskeleton to the nucleus. Nat Rev Mol Cell Biol. 2004;5(11):920-931.

20. Vallee BL. The function of methallothionein. Neurochem Int. 1995;27:23-33.

21. Eide DJ. The SLC39 family of metal ion transporter. Pflugers Arch. 2004;447:796-800.

22. Palmiter RD, Huang L. Efflux and compartmentalization of zinc by members of the SLC30 family of solute carriers. Pflugers Arch. 2004;447(5):744-751.

23. Colvin RA, Fontaine CP, Laskowski M and Thomas D. $\mathrm{Zn} 2+$ transporters and $\mathrm{Zn} 2+$ homeostasis in neurons. Eur J Pharmacol. 2003;479:298-302.

24. Kambe T, Yamaguchi-Iwai Y, Sasaki R and Nagao M. Overview of mammalian zinc transpoters. Cell. Mol. Life Sci. 2004;61:298-302.

25. Ganss B, Jheon A. Zinc finger transcription factors in skeletal development. Crit Rev Oral Biol Med. 2004;15(5):282-297.

26. Licastro F, Mariani RA, Faldella G, Carpene E, Guidicini G, Rangoni A, Grilli T, et al. Immune-endocrine status and coeliac disease in children with Down's syndrome: relationships with zinc and cognitive efficiency. Brain Res Bull. 2001;55(2):313-317.

27. Prasad AS. Zinc and immunity. Mol Cell Biochem. 1998;188(1-2):63-69.

28. Kitamura H, Morikawa H, Kamon H, Iguchi M, Hojyo S, Fukada T, Yamashita S, et al. Toll-like receptor-mediated regulation of zinc homeostasis influences dendritic cell function. Nat Immunol. 2006;7(9):971-977.

29. Chiba M, Katayama K, Takeda R, Morita R, Iwahashi K, Onishi Y, Kita H, et al. Diuretics aggravate zinc deficiency in patients with liver cirrhosis by increasing zinc excretion in urine. Hepatol Res. 2013;43(4):365-373.

30. Van der Rijt CC, Schalm SW, Schat H, Foeken K, De Jong G. Overt hepatic encephalopathy precipitated by zinc deficiency. Gastroenterology. 1991;100(4):1114-1118.

31. Rabbani P, Prasad A. Plasma ammonia and liver ornithine transcarbamylase activity in zinc deficiency in humans. Am J Physiol. 1978;235:E203-E206.

32. Prasad AS, Rabbani P, Abbasii A, Bowersox E, Fox MR. Experimental zinc deficiency in humans. Ann Intern Med. 1978;89(4):483-490.

33. Reding P, Duchateau J, Bataille C. Oral zinc supplementation improves hepatic encephalopathy. Results of a randomised controlled trial. Lancet. 1984;2(8401):493-495.

34. Riggio O, Merli M, Capocaccia L, Caschera M, Zullo A, Pinto G, Gaudio E, et al. Zinc supplementation reduces blood ammonia and increases liver ornithine transcarbamylase activity in experimental cirrhosis. Hepatology. 1992;16(3):785-789.

35. Hayashi M, Ikezawa K, Ono A, Okabayashi S, Hayashi Y, Shimizu S, Mizuno T, et al. Evaluation of the effects of combination therapy with branched-chain amino acid and zinc supplements on nitrogen metabolism in liver cirrhosis. Hepatol Res. 2007;37(8):615-619.

36. Katayama K, Sakakibara M, Imanaka K, Ohkawa K, Matsunaga T, Naito M, Ito T. Effect of zinc supplementation in patients with type $\mathrm{C}$ liver cirrhosis. Open J Gastroenterology. 2011;1:22-28.

37. Riggio O, Ariosto F, Merli M, Caschera M, Zullo A, Balducci G, Ziparo V, et al. Short-term oral zinc supplementation does not improve chronic hepatic encephalopathy. Results of a double-blind crossover trial. Dig Dis Sci. 1991;36(9):1204-1208.

38. Katayama K, Saito M, Kawaguchi T, Endo R, Sawara $\mathrm{K}$, Nishiguchi S, Kato A, et al. Effect of zinc on liver cirrhosis with hyperammonemia: a preliminary randomized, placebo-controlled double-blind trial. Nutrition. 2014;30(11-12):1409-1414.

39. Dodson G, Steiner D. The role of assembly in insulin's biosynthesis. Curr Opin Struct Biol. 1998;8(2):189-194.

40. Begin-Heick N, Dalpe-Scott M, Rowe J, Heick HM. Zinc supplementation attenuates insulin secretory activity in pancreatic islets of the ob/ob mouse. Diabetes. 1985;34(2):179-184.

41. Haase H, Maret W. Protein tyrosine phosphatases as targets of the combined insulinomimetic effects of zinc and oxidants. Biometals. 2005;18(4):333-338.

42. Soinio M, Marniemi J, Laakso M, Pyorala K, Lehto S, Ronnemaa T. Serum zinc level and coronary heart disease events in patients with type 2 diabetes. Diabetes Care. 2007;30(3):523-528.

43. Jayawardena R, Ranasinghe P, Galappatthy P, Malkanthi $\mathrm{R}$, Constantine G, Katulanda P. Effects of zinc supplementation on diabetes mellitus: a systematic review and meta-analysis. Diabetol Metab Syndr. 2012;4(1):13.

44. Holecek M. Ammonia and amino acid profiles in liver cirrhosis: effects of variables leading to hepatic encephalopathy. Nutrition. 2015;31(1):14-20.

45. Pramoolsinsap C, Promvanit N, Komindr S, Lerdverasirikul P, Srianujata S. Serum trace metals in chronic vi- 
ral hepatitis and hepatocellular carcinoma in Thailand. J Gastroenterol. 1994;29(5):610-615.

46. Mendez-Sanchez N, Martinez M, Gonzalez V, RoldanValadez E, Flores MA, Uribe M. Zinc sulfate inhibits the enterohepatic cycling of unconjugated bilirubin in subjects with Gilbert's syndrome. Ann Hepatol. 2002;1(1):40-43.

47. Ranasinghe $\mathrm{P}$, Wathurapatha WS, Ishara MH, Jaya- wardana R, Galappatthy P, Katulanda P, Constantine GR. Effects of Zinc supplementation on serum lipids: a systematic review and meta-analysis. Nutr Metab (Lond). 2015;12:26.

48. Milman N, Hvid-Jacobsen K, Hegnhoj J, Sorensen SS. Zinc absorption in patients with compensated alcoholic cirrhosis. Scand J Gastroenterol. 1983;18(7):871-875. 